1094

\section{CORD BLOOD BRAIN DERIVED NEUROTROPHIC FACTOR: DIAGNOSTIC AND PROGNOSTIC MARKER IN FULLTERM NEWBORNS WITH PERINATAL ASPHYXIA}

doi:10.1136/archdischild-2012-302724.1094

S Atef, G Gad, S Imam, M Shawky. Ain Shams University, Cairo, Egypt

Backgrounds This prospective case control study was designed to evaluate cord blood brain derived neurotrophic factor level in full term newborns with perinatal asphyxia as a marker of central nervous system insult and predictor of severity of hypoxic ischemic encephalopathy, with follow up of its level during the reperfusion phase.

Material and Methods The study included twenty fullterm neonates with perinatal asphyxia (cases) and twenty controls. Cord blood samples were obtained at birth and peripheral blood samples at $72 \mathrm{~h}$ postnatal from cases only. Plasma brain derived neurotrophic factor level was measured using enzyme linked immunosorbent assay. The clinical severity of encephalopathy was graded based on Sarnat and Sarnat staging.

Results Cord Plasma brain derived neurotrophic factor level was significantly increased among cases compared to controls. Among cases, brain derived neurotrophic factor level at delivery and after $72 \mathrm{~h}$ significantly correlated with the severity of encephalopathy according to Sarnat staging being higher as severity increases. Brain derived neurotrophic factor level significantly increased after $72 \mathrm{~h}$ of life compared to its level at delivery among cases. Brain derived neurotrophic factor levels at delivery and at $72 \mathrm{~h}$ postnatal were predictors of severe Sarnat stage and poor outcome.

Conclusion We concluded that brain derived neurotrophic factor level as a marker of central nervous system insult is increased in full term newborns with perinatal asphyxia. It can serve as an indicator for the severity of encephalopathy and adverse outcomes.

\section{USE OF AMPLITUDE INTEGRATED ELECTROENCEPHALOGRAPHY IN NEWBORNS WITH SEVERE HYPERBILIRUBINEMIA}

doi:10.1136/archdischild-2012-302724.1095

M Chang, JH Shin. Department of Pediatrics, Chungnam National University Hospital, Daejeon, Republic of Korea

Background and Aims The spectrum of bilirubin-induced neurologic dysfunction (BIND) is very wide and the symptoms and signs may be very mild or absent. Amplitude-integrated electroencephalography (aEEG) allows continuous trend recording of cerebral function in high-risk newborns. However, published knowledge regarding correlation between aEEG and BIND remains limited.

In this study, we hypothesized that abnormal aEEG in infants with severe hyperbilirubinemia is useful for detection of asymptomatic BIND.

Methods This is a prospective observational study of newborns with severe hyperbilirubinemia in our NICU from April 2011 to December 2011. Patients were included if they were $\geq 34$ weeks gestational age (GA) at birth and their total serum bilirubin [TSB] $>20 \mathrm{mg} / \mathrm{dL}$. The aEEG was performed for 6 hours since admission, and rechecked when TSB is below $10 \mathrm{mg} / \mathrm{dL}$.

Results Fourteen infants were enrolled. Male to female was 10 to 4 . Their GA was $37.2 \pm 1.2$ weeks and their birth weight was $3,238 \pm 421 \mathrm{~g}$. Their peak TSB was $23.63 \pm 2.7 \mathrm{mg} / \mathrm{dL}$. Photopherapies were performed in all infants and exchange transfusions were also performed in 2 infants. Six out of 14 infants (42.8\%) showed abnormal aEEG findings such as discontinuity, abnormal cycling, depressed lower border and abnormal bandwidth span when their TSB were markedly elevated. However none had any noticeable symptoms or signs of neurologic dysfunction. All abnormal aEEG findings were normalized after treatment.
Conclusions Abnormal aEEG finding in infants with severe hyperbilirubinemia is useful for detection of asymptomatic BIND and can be reversible with appropriate treatment.

\section{ENIGMA OF MANAGEMENT OF SEIZURES IN HYPOXIC ISCHEMIC ENCEPHALOPATHY (HIE) - WHEN TO STOP ANTICONVULSANTS?}

doi:10.1136/archdischild-2012-302724.1096

'S Nangia, 'A Saili, ${ }^{2}$ A Garg. 'Division of Neonatology, Department of Pediatrics, ${ }^{2}$ Department of Pediatrics, Lady Hardinge Medical College and Kalawati Saran Children's Hospital, New Delhi, India

Background Although there is some agreement regarding what and when to initiate as anticonvulsant medication for seizures in HIE, there is no consensus about when to stop medication.

Objective To assess the effect of early stoppage of anticonvulsant drugs in HIE on seizure recurrence and neurological outcome.

Design/methods This prospective study enrolled neonates with HIE with non-metabolic seizures from August 2007 to July 2010. A loading dose of $20 \mathrm{mg} / \mathrm{kg}$ of phenobarbitone was used for seizure control. Additional mini-boluses of $5 \mathrm{mg} / \mathrm{kg}$ till a cumulative dose of $40 \mathrm{mg} / \mathrm{kg}$ followed by phenytoin was used if required.

Results Out of 59 neonates, $85 \%$ had cord pH below 7.2 and $83 \%$ had $\mathrm{BE}$ of -12 or higher. At birth $89 \%$ required positive pressure ventilation and $40 \%$ needed mechanical ventilation during NICU stay. 26/59(44\%) had seizure onset before $6 \mathrm{hrs}$ and 23/59 (39\%) between $6-12 \mathrm{hrs}$. $64 \%$ had a single episode of seizure, $22 \%$ had $2-3$ episodes and 10\% had 4-6 episodes. Twelve babies expired and 47 were discharged without anticonvulsant. There was no recurrence of seizure in $44 / 47(94 \%)$. On follow up at 3 months $40 / 47(85 \%)$, at 6 months $30 / 35(86 \%)$, at 12 months $25 / 29(86 \%)$, at 24 months $23 / 26(89 \%)$ and at 30 months $13 / 16(81 \%)$ had normal neurological outcome.

Conclusions This pilot work suggests that potentially apoptotic anticonvulsant drugs can probably be stopped early as soon as seizures abate in HIE without increased risk of seizure recurrence or adverse neurological outcome.

\section{ACTIVATED PROTEIN C DECREASES ENDOTOXIN-INDUCED INFLAMMATORY RESPONSES IN INFANTS WITH NEONATAL ENCEPHALOPATHY}

doi:10.1136/archdischild-2012-302724.1097

1,2,3,4 HO Eliwan, 2,3FO O'Hare, ${ }^{2} \mathrm{D}$ Sweetman, ${ }^{3} \mathrm{~W}$ Watson, ${ }^{3} \mathrm{~A}$ O'Neill, ${ }^{1,4,5,6} \mathrm{EJ}$ Molloy. ${ }^{1}$ Neonatology, Our Lady's Children's Hospital, Crumlin; ${ }^{2}$ Neonatology, National Maternity Hospital, Holles Street; ${ }^{3}$ UCD School of Medicine and Medical Science, Conway Institute for Biomolecular and Biomedical Science, University College Dublin; ${ }^{4}$ Royal College of Surgeon; ${ }^{5}$ National Maternity Hospital, Holles Street; ${ }^{6}$ UCD School of Medicine and Medical Science, Conway Institute for Biomolecular and Biomedical Science, Univeristy College Dublin, Dublin, Ireland

Introduction Infection and inflammation can be antecedents of neonatal encephaloapthy (NE) and increase the risk of neurological sequelae. Activated protein C (APC) has anticoagulant and antiinflammatory effects and provides neuroprotection in ischemic brain and spinal cord injury.

Aims To examine neutrophil and monocyte responses to Lipopolysaccharide (LPS) in infants with NE $(n=22)$ and also the effect of APC compared with healthy adult controls $(n=15)$.

Methods Whole blood was incubated with LPS +/-APC and TLR4, $\mathrm{CD} 11 \mathrm{~b}$ expression, and reactive oxygen intermediate (ROI) release from neutrophils and monocytes was examined by flow cytometry.

Results Neutrophil and monocyte CD11b expression was significantly increased in response to LPS in adults controls $(p<0.001)$ and NE infants $(p<0.001)$. However infants with NE were LPS-hyporesponsive 
compared to adults control and APC did not reduce this effect. Neutrophil TLR4 expression was significantly increased in response to LPS in NE infants on D3 compared to adults $(p<0.001)$ and has been reduced by APC ( $p=0.03)$. LPS induced monocyte TLR4 was only significantly increased in NE infants D7 $(p<0.001)$. Neutrophil ROI was significantly increased in Adults $(p<0.001)$ and NE infants on D3 ( $p=0.021)$ following LPS and this response were significantly reduced by APC.

Conclusion Neutrophil activation and production of ROI may mediate tissue damage in NE infants. APC modified LPS responses in adults and NE infants on D3 of life. APC may reduce the inflammatory responses secondary to hypoxia and possibly benefit these patients at high risk of inflammatory multiorgan dysfunction.

\section{CARDIAC OUTPUT MEASUREMENTS IN PRETERM NEONATES REQUIRING RESUSCITATION AT BIRTH}

doi:10.1136/archdischild-2012-302724.1098

N Ahmed, V Sundaram, P Kumar. PGIMER, Chandigarh, India

Background The effect of perinatal asphyxia on cardiac output and flow patterns in asphyxiated preterm neonates is less well understood.

Objectives To study the cardiac outputs (left and right ventricleLVO and RVO) and superior vena cava (SVC) blood flow patterns in asphyxiated preterm neonates in first 24 hours of age.

Subject and Interventions Serial echocardiography was done in preterm neonates $<34$ weeks who required resuscitation, at $6 \pm 2$, $12 \pm 2$ and $24 \pm 4$ hours using color Doppler (Sonosite). LVO, RVO and SVC flow velocity were calculated

Results Functional Echo was done in 68 neonates with mean gestation and weight of $31 \pm 1.6$ weeks and $1343 \pm 361 \mathrm{~g}$. Median SVC flow, LVO and RVO at 6, 12 and 24 hrs of age were 109 (70-137), 103 (85-150) and 132 (92-181); 381 (287-493), 421 (337-510) and 408 (324-557); 327 (214-435), 328 (259-467) and 381 (280-501) $\mathrm{ml} / \mathrm{kg} / \mathrm{min}$ respectively. The differences in these three measures between three time points were not statistically significant. A statistically significant increase was seen between SVC flows at 6 versus 24 hours. No difference was observed in these measurements in $21 \%$ vs $100 \%$ oxygen groups.

Conclusions LVO, RVO and SVC flow showed an increasing trend from 6 hrs of age to 24 hrs of age. A significant increase was observed in the SVC flow between 6 and 24 hours of age suggestive of hypoperfusion-reperfusion phenomena. Resuscitating with $21 \%$ or $100 \%$ oxygen did not show any difference in these measurements.

\section{NORMATIVE LEVELS OF INTERLEUKIN 16 IN UMBILICAL CORD BLOOD}

doi:10.1136/archdischild-2012-302724.1099

NM Denihan, AM Looney, GB Boylan, BH Walsh, DM Murray. Neonatal Brain Research Group, Department of Paediatrics and Child Health, Cork University Maternity Hospital, Cork, Ireland

Background and Aims The need for early and accurate prediction of outcome in Hypoxic-Ischemic Encephalopathy (HIE) remains critical. We have previously demonstrated that Interleukin 16 (IL-16) is raised in the umbilical cord blood of infants with moderate and severe HIE and has the potential to be developed as a predictive biomarker. Normal reference ranges for IL-16 in umbilical cord blood have not been previously described. The aim of this study was to determine normative levels of IL-16 in full term neonates using cord blood following uncomplicated deliveries.

Methods Full term infants were recruited as part of an ongoing birth cohort study, the Cork BASELINE Birth Cohort Study. All had cord blood drawn and bio-banked at $-80^{\circ} \mathrm{C}$, within 3 hours of birth. Samples were chosen based on Apgar scores ( $\geq 8$ at $1 \mathrm{~min}, \geq 9$ at $5 \mathrm{~min})$, duration of ruptured membranes $<24 \mathrm{~h}$, temperature in labour $\leq 37^{\circ} \mathrm{C}$, gestational age $\geq 37$ weeks and birthweight centile $\geq 10 \%$. Analysis was performed on plasma EDTA, using ELISA Quantikine ${ }^{\circledR}$ (R\&D Systems, Europe).

Results The study consisted of samples from 48 infants with two different modes of delivery; unassisted vaginal delivery ( $\mathrm{n}=12$ male, $\mathrm{n}=12$ female) and pre-labour elective caesarean section ( $\mathrm{n}=12$ male, $\mathrm{n}=12$ female). The range of all samples was normally distributed between 87.0 and $114.6 \mathrm{pg} / \mathrm{ml}$. Mean (SD) for IL-16 was 103.1 $( \pm 21.9) \mathrm{pg} / \mathrm{ml}$. Levels were not affected by gender or mode of delivery.

Conclusion For the first time we have described the expected range of cord plasma IL-16 levels in healthy term infants.

\section{C-REACTIVE PROTEIN CONCENTRATIONS IN NEONATES WITH HYPOXIC-ISCHAEMIC ENCEPHALOPATHY AND EFFECT OF TOTAL BODY HYPOTHERMIA}

doi:10.1136/archdischild-2012-302724.1100

'S Sanka, 'H Muniraman, ${ }^{2} \mathrm{D}$ Gardner, ${ }^{2} \mathrm{~A}$ Pawaletz, ${ }^{3} \mathrm{C}$ Jennings, ${ }^{3} \mathrm{~A}$ Vayalakkad, ${ }^{3,4} \mathrm{~S}$ Victor, ${ }^{2.5} \mathrm{MA}$ Turner, 'P Clarke. 'Neonatal Unit, Norfolk and Norwich University Hospitals NHS Foundation Trust, Norwich; ' 2 Neonatal Unit, Liverpool Women's Hospital, Liverpool; ${ }^{3}$ Newborn Intensive Care Unit, Central Manchester University Hospitals NHS Foundation Trust; ${ }^{4}$ Developmental Biomedicine Research Group, University of Manchester, Manchester; ${ }^{5}$ Department of Women's and Children's Health, Institute of Translational Medicine, University of Liverpool, Liverpool, UK

Background and Aims Production of C-reactive protein (CRP), an acute phase reactant of hepatic origin, may be affected by perinatal asphyxia. This study tested hypotheses that circulatory CRP concentrations correlate with clinical severity of hypoxic-ischaemic encephalopathy (HIE) and that total body hypothermia modulates CRP response.

Methods Clinical records in three centres were reviewed for neonates $\geq 36$ weeks' gestation admitted between 01/07/06 and $30 / 06 / 11$ with HIE of any severity (grades 1-3 Sarnat-Sarnat). Participating centres adopted routine cooling at different dates. Data extracted included CRP concentrations in the first postnatal week measured during routine clinical practice, clinical HIE grading, and reception of therapeutic hypothermia. Proportions with raised CRP $(>10 \mathrm{mg} / \mathrm{L})$, and maximum CRP concentrations were assessed according to HIE grade and whether cooled.

Results A raised CRP was present in 150/259(58\%) neonates during the first postnatal week (HIE1: 30/73[41\%], HIE2: 83/129[64\%], HIE3: $37 / 57[65 \%$ ], $\mathrm{p}=0.003$ ) but elevated maximum concentrations (peaking median day 3 ) did not differ between HIE grades (median [range] HIE1: 31.3 [10.0-188.1] mg/L, HIE2: 32.5 [10.0-305.9] mg/L, HIE3: 34.0 [10.2-346.5] mg/L, $\mathrm{p}=0.48)$. A raised CRP was present in $117 / 187(63 \%)$ cooled and $33 / 72(46 \%)$ non-cooled infants ( $p=0.02)$, but their peak CRP concentrations did not differ (median [range] CRP cooled vs. non-cooled: 31.9 [10.0-346.5] mg/L vs. 53.0 [10.4188.1] mg/L, $\mathrm{p}=0.26$ ).

Conclusion A raised CRP is a common finding in the first postnatal week in neonates admitted with HIE and is found in most infants with moderate-severe HIE. Peak CRP concentrations did not differ with clinical HIE grade and whole body hypothermia did not significantly affect peak CRP concentrations.

\section{ARE LACTAT DEHYDROGENASE AN.D NEURON SPECIFIC ENOLASE ANALYSES GOOD DIAGNOSTIC TOOLS FOR ASSESSING EXTENSION OF PERINATAL HYPOXIC- ISCHEMIC BRAIN INJURY?}

doi:10.1136/archdischild-2012-302724.1101

${ }^{1} \mathrm{~B}$ Vasiljevic, ${ }^{2} \mathrm{~S}$ Maglajic, ${ }^{3} \mathrm{M}$ Gojnic, ${ }^{4} \mathrm{D}$ Lutovac, ${ }^{5} \mathrm{D}$ Bogicevic. ${ }^{1}$ Neonatology, Institute of Gynecology and Obstetrics, Clinical Centre of Serbia; ${ }^{2}$ Neonatology, University Children's Hospital; ${ }^{3}$ Perinatology, Institute of Gynecology and Obstetrics, Clinical Centre 